\title{
High temperature $X$-ray diffraction study of enstatite up to the melting point
}

\author{
Dayong JiAnG*, Kiyoshi FuJINO*, Naotaka TOMIOKA**, \\ Tomohiko HosoyA* and Kaushik Das*** \\ *Division of Earth and Planetary Sciences, Graduate School of Science, \\ Hokkaido University, Sapporo 060-0810, Japan \\ **Department of Earth and Planetary Sciences, Kobe University, Kobe 657-8501, Japan \\ ***Advanced Materials Laboratory, National Institute for Materials Science, Ibaraki 305-0044, Japan
}

\begin{abstract}
In order to examine the phase relations of enstatite at high temperatures, high temperature $\mathrm{X}$-ray powder diffraction experiments were performed with low clinoenstatite $\mathrm{MgSiO}_{3}$ as starting materials. It was observed that upon heating low clinoenstatite transformed into protoenstatite at around $1100^{\circ} \mathrm{Cand}$ protoenstatite persisted up to the melting point, indicating that protoenstatite is the high temperature stable phase for $\mathrm{MgSiO}_{3}$ up to the melting point. Two extra peaks $\left(d=3.074-3.079 \AA\right.$ and $3.323-3.331 \AA$ at $\left.1200^{\circ} \mathrm{C}\right)$ which could not be indexed as low clinoenstatite or protoenstatite, appeared along with protoenstatite at around $1150^{\circ} \mathrm{C}$, but they disappeared at temperatures above $\sim 1400^{\circ} \mathrm{C}$. These extra peaks did not reappear on cooling of protoenstatite. This indicates that these extra peaks originate from high temperature clinoenstatite metastably transformed from low clinoenstatite and that high temperature clinoenstatite has no stability field above and below that of protoenstatite.
\end{abstract}

\section{Introduction}

Polymorphism of enstatite $\mathrm{MgSiO}_{3}$ has been extensively studied by many investigators. Five different structure forms have been known; orthoenstatite (OEn) with space group Pbca, protoenstatite (PEn) with Pbcn, low clinoenstatite (CEn) with $\mathrm{P}_{1} / \mathrm{c}$, high temperature clinoenstatite (high-T CEn) with $\mathrm{C} 2 / \mathrm{c}$ and high pressure clinoenstatite (high-P CEn) with $\mathrm{C} 2 / \mathrm{c}$. The stable phase of enstatite at ambient pressure and temperature is generally thought to be CEn, and OEn becoming stable above $\sim 600^{\circ} \mathrm{C}$. PEn appears to be stable from $\sim 1000^{\circ} \mathrm{C}$ to the incongruent melting point $\sim 1557^{\circ} \mathrm{C}$ as indicated by Kushiro (1972) and Smyth (1974). With increasing pressure, high-P CEn becomes stable above $\sim 6 \mathrm{GPa}$ at room temperature (Angel et al., 1992).

The controversy is about the appearance and stability of high-T CEn. High-T CEn indexed with a triclinic cell was first reported to appear during the CEn-PEn inversion by Perrotta and Stephenson (1965), and then

K. Fujino, fujino@ep.sci.hokudai.ac.jp Corresponding author N. Tomioka, nao@kobe-u.ac.jp

K. Das, kaushik.das@ nims.go.jp its diffraction patterns were reindexed by Smith (1969) with a monoclinic cell belonging to space group $\mathrm{C} 2 / \mathrm{c}$. The subsequent studies showed much controversy. Sadanaga and Okamura (1971), Shimobayashi and Kitamura (1991) and Iishi and Kitayama (1995) reported the appearance of high-T CEn at high temperatures above $\sim 1000^{\circ} \mathrm{C}$. Smyth (1969) reported that orthopyroxene with a composition $\left(\mathrm{Mg}_{0.3} \mathrm{Fe}_{0.7}\right) \mathrm{SiO}_{3}$ was also transformed into the high temperature form with $\mathrm{C} 2 / \mathrm{c}$ at $1050^{\circ} \mathrm{C}$. Further, Shimobayashi and Kitamura (1993) observed high- $\mathrm{T}$ CEn above $\sim 1200^{\circ} \mathrm{C}$ using $\mathrm{MgSiO}_{3}$ OEn as a starting material by high temperature transmission electron microscopy (HTTEM). However, high-T CEn was not observed in the transition between CEn and PEn by Sarver and Hummel (1962) and Smyth (1974), nor in the transition between OEn and PEn by Sadanaga et al. (1969), Murakami et al. (1982), Boysen et al. (1991) and Yang and Ghose (1995).

Several reports concerning the occurrence of other intermediate structures also made the problem complicated. Ernst and Schwab (1970) reported a new high temperature phase between $\mathrm{CEn}$ and PEn, very similar to $\mathrm{PEn}$ and stable between $1080-1260{ }^{\circ} \mathrm{C}$ with a characteristic peak of $d=3.06 \AA$ at $1145^{\circ} \mathrm{C}$. Murakami et al. 
(1982) observed that with increasing temperature the OEn-PEn inversion is preceded by the occurrence of an intermediate structure in which the silicate chains are stretched like those of PEn but the structural scheme of OEn is preserved. Schrader et al. (1990) gave a similar explanation for the intermediate structure they observed. Yang and Ghose (1995) also reported some precursor phenomena in the OEn-PEn transition in which weak satellite reflections of uncertain origin appeared at the lower angle side of a few reflections. High-T CEn and these intermediate structures seem to have some features in common that appear during the OEn/CEn-PEn transition.

Meanwhile, Gasparik (1990) predicted from the thermodynamic calculation that there is a narrow stability field for high-T CEn above PEn and below the melting point. With the molecular dynamic simulation Matsui and Price (1992) proposed a high temperature orthorhombic phase of $\mathrm{MgSiO}_{3}$ with space group Bmcm also stable between PEn and the melting point. However, Smyth (1974) did observe neither high-T CEn $(\mathrm{C} 2 / \mathrm{c})$ phase nor high temperature orthorhombic phase $(\mathrm{Bmcm})$ in the high temperature single crystal $\mathrm{X}$-ray study up to the melting point.

All of the above arguments indicate that the phase relations of enstatite polymorphs, particularly of high-T CEn at high temperature are still not clarified yet. The present study intends to elucidate the high temperature phase relations of enstatite by high temperature in situ $\mathrm{X}$-ray diffraction experiments up to the melting point.

\section{Experimental procedures}

\section{Starting materials}

The starting materials used for this study were synthetic $\mathrm{MgSiO}_{3} \mathrm{CEn}$ obtained by heating a $1: 1$ mole ratio mixture of $\mathrm{MgO}$ and $\mathrm{SiO}_{2}$. In preliminary heating syntheses at $1300-1500{ }^{\circ} \mathrm{C}$, the quenched samples showed mixed X-ray peaks of CEn and PEn. However, the quenched samples heated at $1550^{\circ} \mathrm{C}$ for three days, showed pure CEn peaks.

\section{High temperature $\mathbf{X}$-ray diffraction experiments}

A high temperature $\mathrm{X}$-ray powder diffractometer (Mac Science Co. Ltd.) was used to examine the phase relations of enstatite at high temperature up to the melting point. The reason to use powdered CEn crystals as starting materials is that the transition rate of powdered crystals is faster than that of a single crystal and CEn transforms faster than OEn. The powdered samples of
Table 1. Sequence of heating steps of the second run

\begin{tabular}{ccccc}
\hline Step & $\begin{array}{c}\text { Temp. } \\
\left({ }^{\circ} \mathrm{C}\right)\end{array}$ & $\begin{array}{c}\text { Standby } \\
\text { time } \\
(\mathrm{min})\end{array}$ & $\begin{array}{c}\text { Changing } \\
\text { Rate* } \\
\left({ }^{\circ} \mathrm{C} / \mathrm{min}\right)\end{array}$ & $\begin{array}{c}\text { Data } \\
\text { collection } \\
\text { time }(\mathrm{min})\end{array}$ \\
\hline 1 & 25 & 0 & 15 & 60 \\
2 & 1000 & 30 & 10 & 60 \\
3 & 1100 & 30 & 10 & 60 \\
4 & 1200 & 30 & 10 & 60 \\
5 & 1250 & 30 & 10 & 60 \\
6 & 1300 & 30 & 10 & 60 \\
7 & 1350 & 30 & 10 & 60 \\
8 & 1400 & 30 & 10 & 60 \\
9 & 1450 & 30 & 10 & 60 \\
10 & 1400 & 30 & 10 & 60 \\
11 & 1350 & 30 & 10 & 60 \\
12 & 1300 & 30 & 10 & 60 \\
13 & 1250 & 30 & 10 & 60 \\
14 & 1200 & 30 & 10 & 60 \\
15 & 1100 & 30 & 10 & 60 \\
16 & 1000 & 30 & 15 & 60 \\
17 & 25 & 0 & & 60 \\
\hline
\end{tabular}

*The changing rate means that temperature was changed with this rate from this step to the next.

CEn were placed onto a Pt holder which was inserted in a $\mathrm{Pt}$-heater placed in the $\mathrm{X}$-ray goniometer. Temperature was monitored by inserting a PR6-30 thermocouple into the hole at the side of the holder. The uncertainty of the measured temperature is considered to be within $10^{\circ} \mathrm{C}$.

Four series of high temperature $\mathrm{X}$-ray diffraction experiments with different heating processes were carried out; run 1: $25-1300^{\circ} \mathrm{C}$, run 2: $25-1450^{\circ} \mathrm{C}$, run 3: $25-1520^{\circ} \mathrm{C}$ and run 4: $25-1550^{\circ} \mathrm{C}$. To show the whole heating process, the sequence of heating steps of the second run is presented in Table 1 as an example, and the whole heating processes of these four runs are illustrated in Figure 1. In all series of runs, the heating and cooling rates of $10^{\circ} \mathrm{C} / \mathrm{min}$ or $15^{\circ} \mathrm{C} / \mathrm{min}$ were adopted and $\mathrm{X}$-ray diffraction data were collected mostly at intervals of $50^{\circ} \mathrm{C}$ or $100^{\circ} \mathrm{C}$ except for some selected temperature ranges where the data were collected at intervals of 10 or $20^{\circ} \mathrm{C}$. Temperatures of the furnace were kept constant for 30 or 60 minutes before the $\mathrm{X}$-ray data were collected. $\mathrm{X}$-ray diffraction intensities were measured with a $2 \theta$ scanning rate of $1^{\circ} / \mathrm{min}$ over the $2 \theta$ range of $10^{-}-70^{\circ}$ for run 1 and run 2 , and of $12-47^{\circ}$ for run 3 and run 4 , respectively. Ni-filtered $\mathrm{CuK} \alpha$ radiation was generated from a rotating anode with the excitation condition of $40 \mathrm{kV}$ and $300 \mathrm{~mA}$. Lattice parameters of CEn and PEn were obtained by the leastsquares fitting by the Wilson \& Pike method using 1840 peaks. 


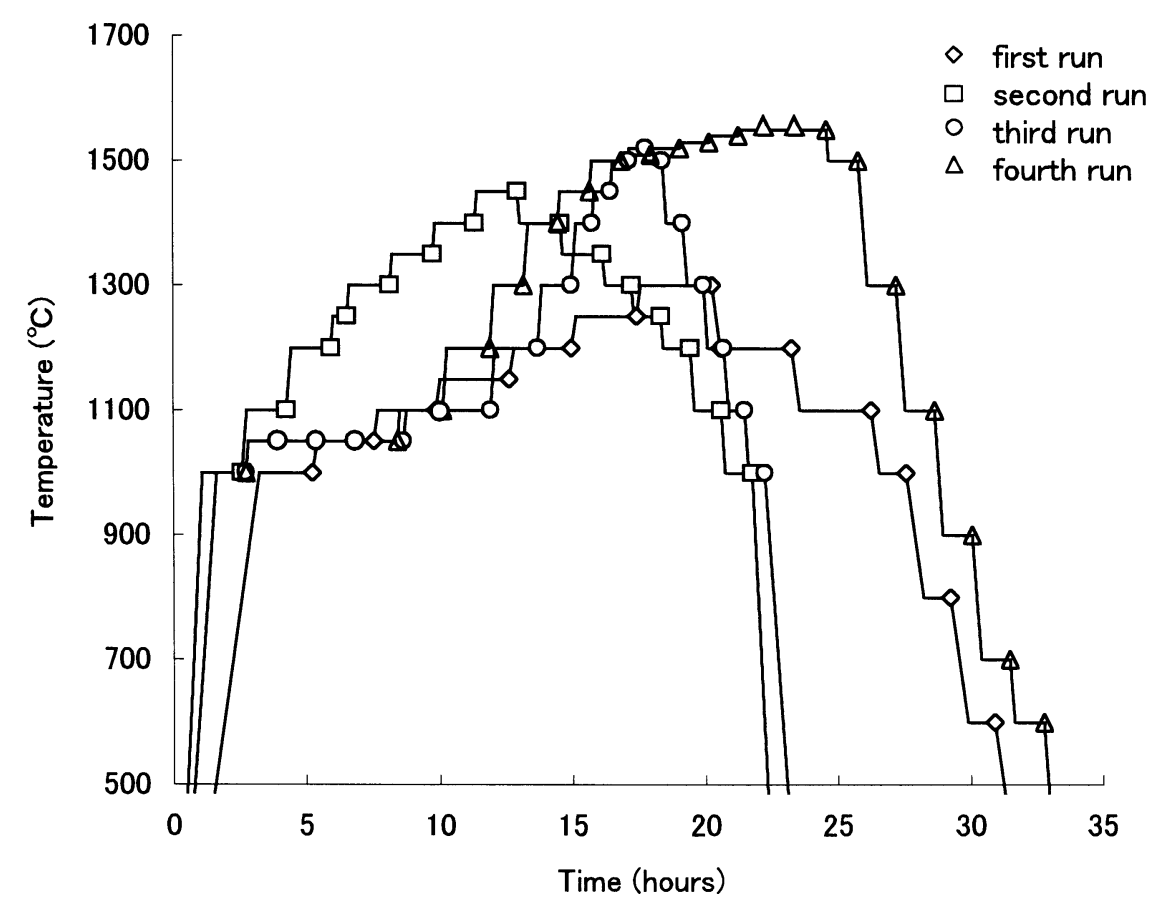

Figure 1. Whole heating processes of the four series of high temperature $\mathrm{X}$-ray experiments. All the symbols mean that diffraction data were collected at those points.

\section{Results}

Variations of the X-ray diffraction profiles of the four runs with increasing and decreasing temperature are illustrated in Figures 2 and 3, respectively. Figure 2a shows the variation of the $\mathrm{X}$-ray diffraction peaks with increasing temperature for the first run. The peaks of $\mathrm{CEn}$ at 1000,1050 and $1100^{\circ} \mathrm{C}$ were indexed by comparing the observed peaks with simulated ones using the structure data by Pannhorst and Mainz (1984). Similarly, the peaks of PEn at and above $1100^{\circ} \mathrm{C}$ were indexed by the same way. The necessary structure data to obtain the simulated pattern was furnished from Yang and Ghose (1995). The observed and calculated $\mathrm{d}^{-}$ values of $\mathrm{CEn}$ at $1000^{\circ} \mathrm{C}$ and of $\mathrm{PEn}$ at $1150^{\circ} \mathrm{C}$ in the first run are summarized in Table 2. In the first run up to $1300^{\circ} \mathrm{C}$, PEn peaks began to appear among those of $\mathrm{CEn}$ at $1100^{\circ} \mathrm{C}$ upon heating, and $\mathrm{CEn}$ disappeared at $1150^{\circ} \mathrm{C}$ (Fig. 2a). At $1100^{\circ} \mathrm{C}$ an extra peak $(\mathrm{d}=$ $3.073 \AA$ ) appeared and further at $1150^{\circ} \mathrm{C}$ another extra peak $(\mathrm{d}=3.332 \AA)$ was recognized. They could not be indexed as PEn or CEn, and they persisted up to the highest setting temperature $1300^{\circ} \mathrm{C}$.

In the second run up to $1450^{\circ} \mathrm{C}$, only CEn peaks were observed up to $1100^{\circ} \mathrm{C}$. But they were replaced by PEn peaks at $1200^{\circ} \mathrm{C}$ (Fig. $2 \mathrm{~b}$ ). Among the PEn peaks two extra peaks which are the same with those found in the first run were also recognized at $1200^{\circ} \mathrm{C}$, and they survived up to the highest temperature $1450^{\circ} \mathrm{C}$. With decreasing temperature in the first and second runs (Fig. $3 \mathrm{a}, \mathrm{b})$, the two extra peaks disappeared at $1000^{\circ} \mathrm{C}$ in the first run and almost disappeared in the second run, while PEn peaks were recognized at $1000^{\circ} \mathrm{C}$ in the second run and even at $600^{\circ} \mathrm{C}$ in the first run.

In the third run up to $1520^{\circ} \mathrm{C}$ and the fourth run up to $1550^{\circ} \mathrm{C}$, very near to the melting point, $\mathrm{PEn}$ and two extra peaks appeared between $1100^{\circ} \mathrm{C}$ and $1200^{\circ} \mathrm{C}$ (Fig. $2 \mathrm{c}, \mathrm{d}$ ). But these extra peaks disappeared above $1400^{\circ} \mathrm{C}$ in the third run and $1500^{\circ} \mathrm{C}$ in the fourth run, respectively, and above those temperatures PEn was the only existing phase. In the cooling processes of PEn, there was no reappearance of the two extra peaks, and PEn peaks still survived below $1000^{\circ} \mathrm{C}$ in the same way as in the first and second runs (Fig. 3c, d).

The lattice parameters of CEn and PEn in the cases of increasing and decreasing temperatures in the second run are listed in Table 3 and the variations of lattice parameters with temperature are illustrated in Figure 4.

\section{Discussion}

\section{Origin of the two extra peaks}

It is clear from the present study that CEn inverts into PEn above around $1100^{\circ} \mathrm{C}$ with increasing temperature and PEn persists up to the melting point, while the two extra peaks appear along with $\mathrm{PEn}$ at around $1150^{\circ} \mathrm{C}$ but disappear above $1400-1500^{\circ} \mathrm{C}$. The problem will 


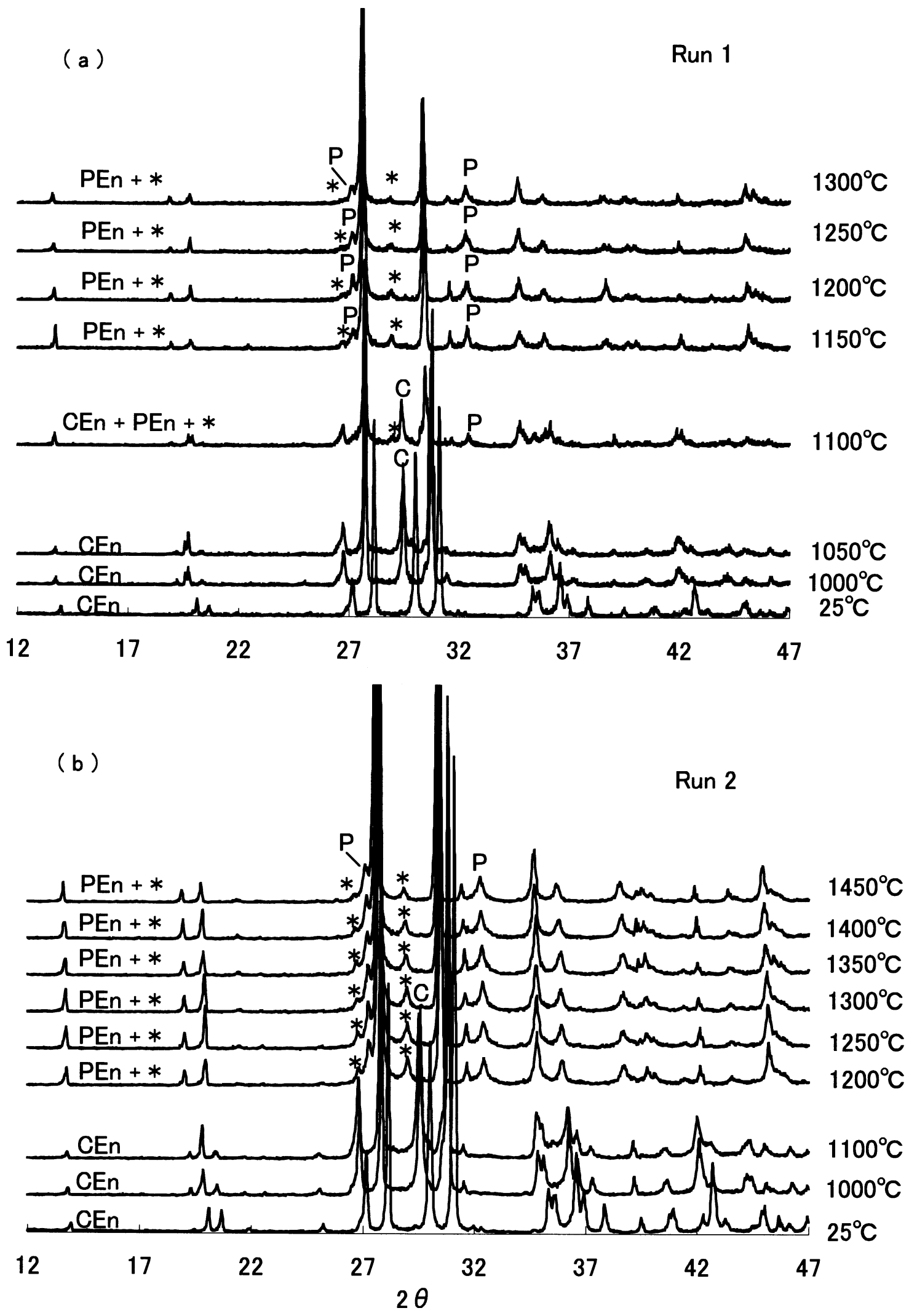

Figure 2. High temperature $\mathrm{X}$-ray powder diffraction patterns of $\mathrm{MgSiO}_{3}$ enstatite for the processes of increasing temperature in (a) the first run, (b) the second run, (c) the third run, and (d) the fourth run. C, the characteritic peak (-221) of CEn; P, the characteristic peaks (211) and (221) of PEn; $*$, the extra peaks which can not be indexed as CEn or PEn. 

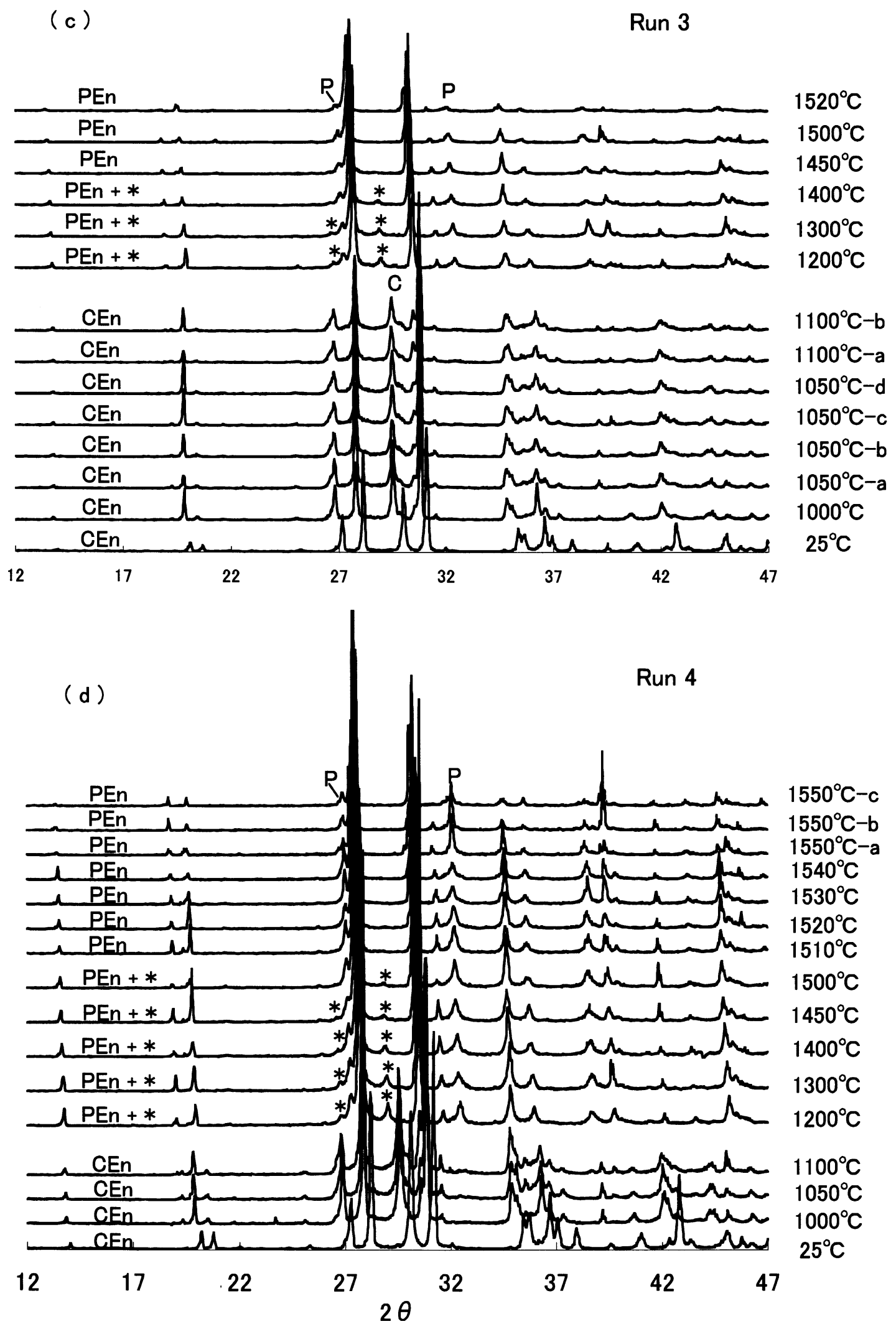

Figure 2. Continued 


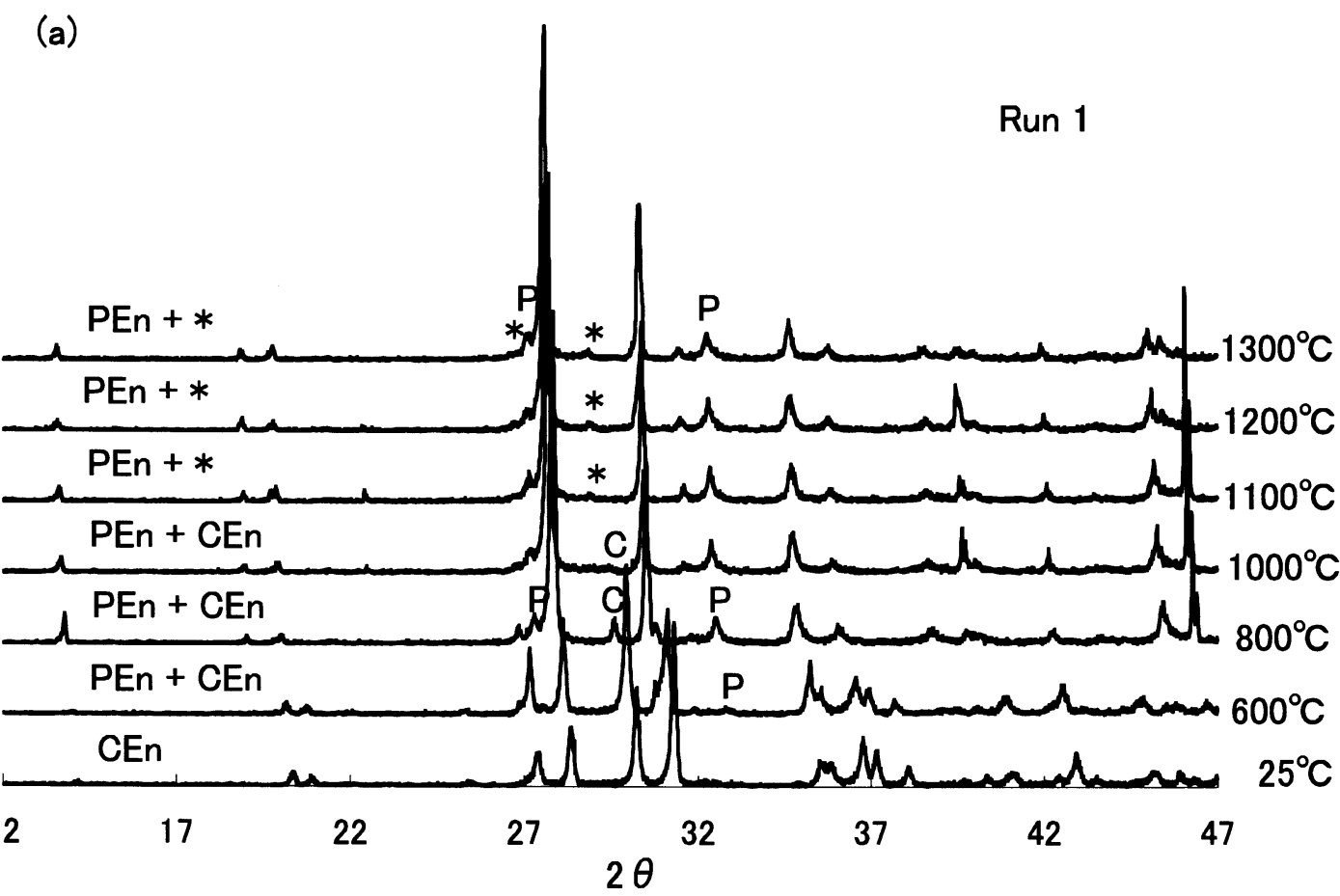

(b)

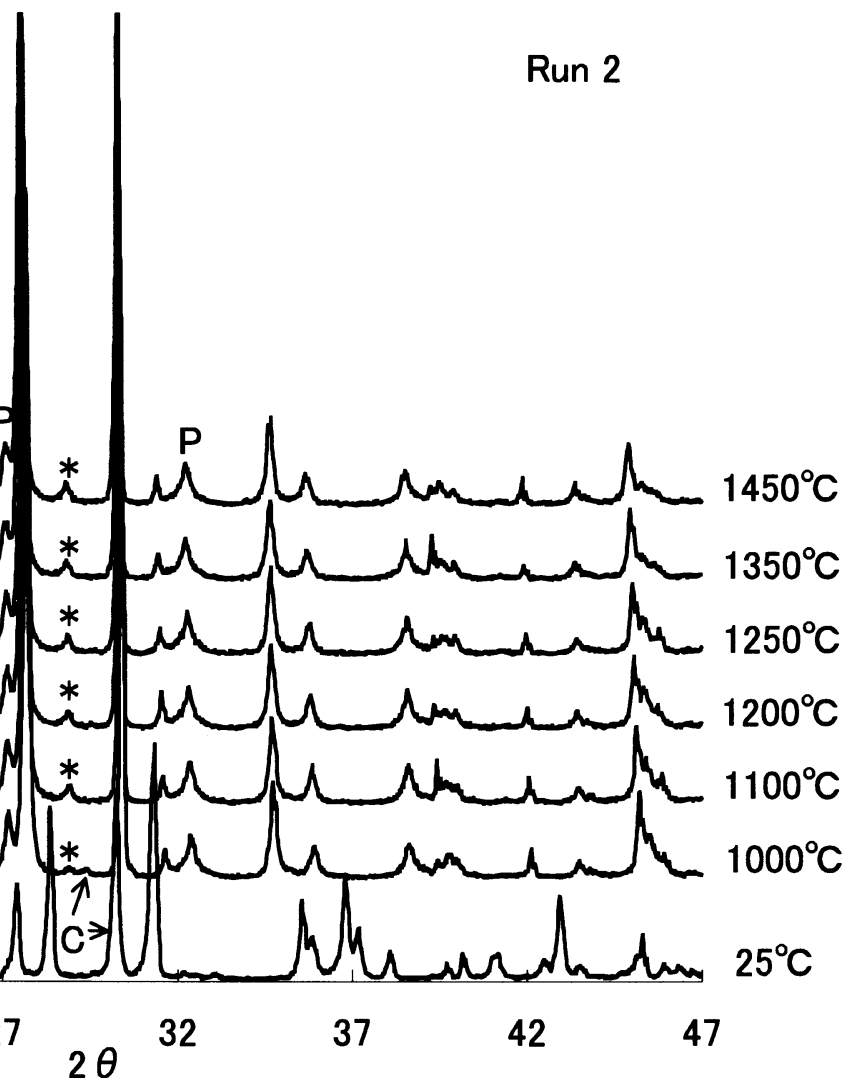

Figure 3. High temperature $\mathrm{X}$-ray powder diffraction patterns of $\mathrm{MgSiO}_{3}$ enstatite for the processes of decreasing temperature in (a) the first run, (b) the second run, (c) the third run, and (d) the fourth run. C, the characteristic peak $(-221)$ of CEn; P, the characteristic peaks (211) and (221) of PEn; $*$, the extra peaks which can not be indexed as CEn or PEn. 


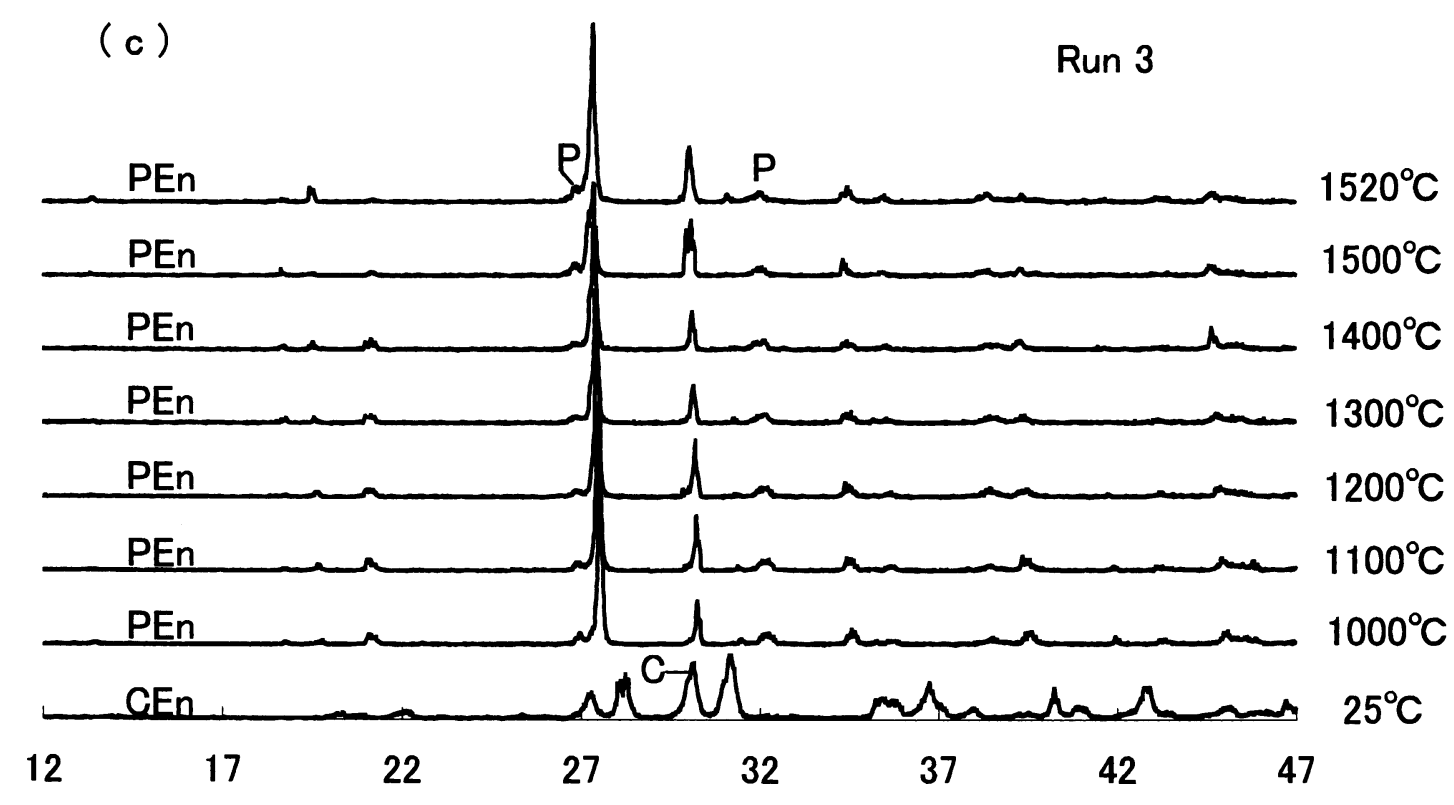

(d)

Run 4

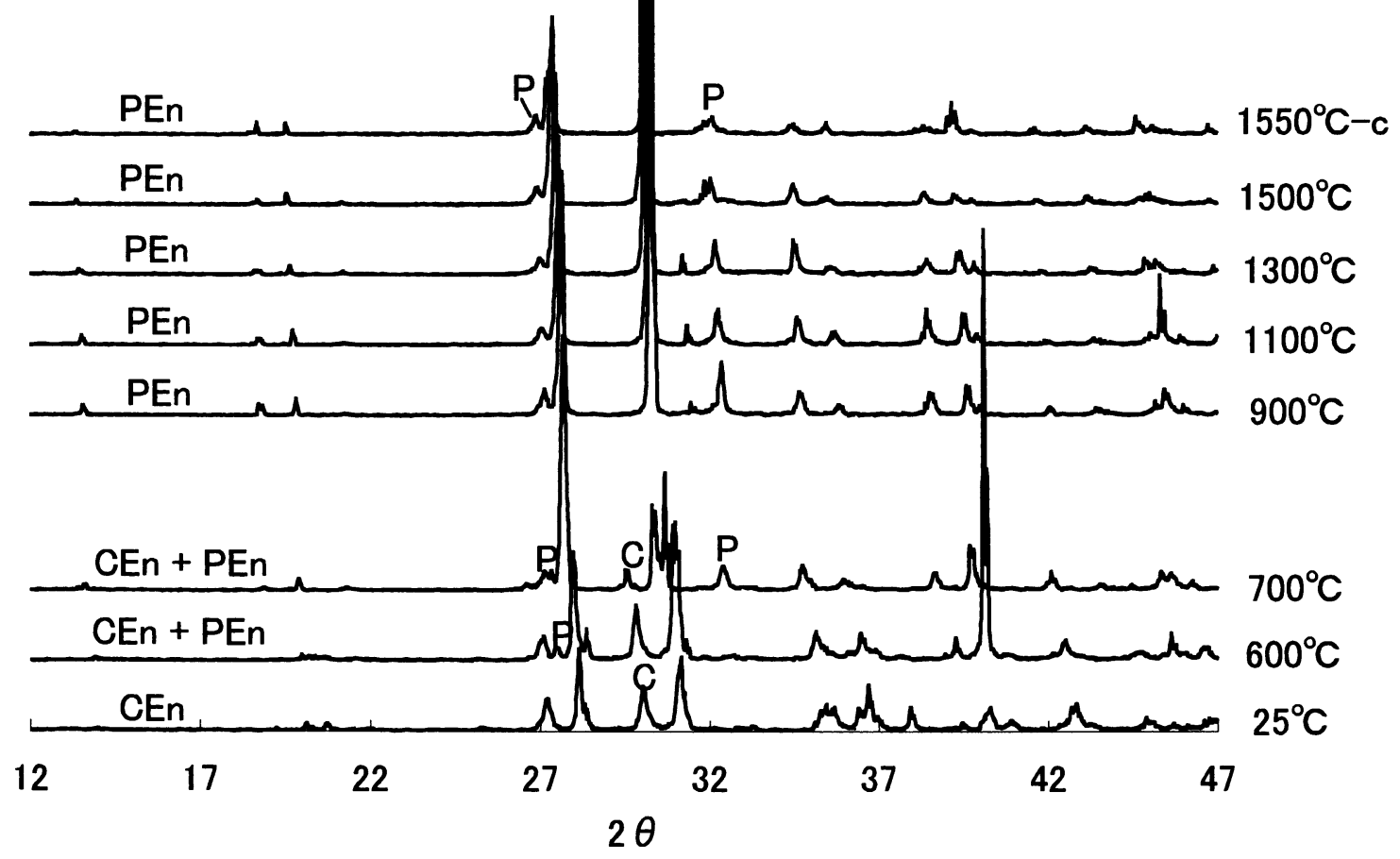

Figure 3. Continued 
Table 2. X-ray diffraction data of low clinoenstatite (CEn) and protoenstatite (PEn) in the first run

CEn at $1000^{\circ} \mathrm{C}$

\begin{tabular}{|c|c|c|c|c|c|c|c|c|c|}
\hline $\mathrm{hkl}$ & $2 \theta\left(^{\circ}\right)$ & $\mathrm{d}_{\mathrm{obs}}(\AA)^{\mathrm{a}}$ & $\mathrm{d}_{\mathrm{cal}}(\AA)^{\mathrm{b}}$ & $\mathrm{I} / \mathrm{I}_{0}$ & hkl & $2 \theta\left(^{\circ}\right)$ & $\mathrm{d}_{\mathrm{obs}}(\AA)^{\mathrm{a}}$ & $\mathrm{d}_{\mathrm{cal}}(\AA)^{\mathrm{c}}$ & $\mathrm{I} / \mathrm{I}_{0}$ \\
\hline 110 & 13.73 & 6.3933 & 6.4142 & 1.5 & 110 & 13.70 & 6.3907 & 6.4297 & 7.9 \\
\hline 200 & 19.22 & 4.5938 & 4.6011 & 1.3 & 200 & 18.94 & 4.6526 & 4.6552 & 2.3 \\
\hline 020 & 19.74 & 4.4751 & 4.4727 & 4.4 & 020 & 19.81 & 4.4522 & 4.4450 & 3.1 \\
\hline 021 & 26.74 & 3.3229 & 3.3203 & 10.6 & & $26.63^{d}$ & 3.3322 & & 1.4 \\
\hline 220 & 27.73 & 3.2070 & 3.2071 & 71.9 & 211 & 27.16 & 3.2687 & 3.2679 & 3.8 \\
\hline-221 & 29.46 & 3.0232 & 3.0231 & 41.2 & 121 & 27.65 & 3.2123 & 3.2110 & 100 \\
\hline 310 & 30.75 & 2.8996 & 2.9015 & 100.0 & & $28.92^{d}$ & 3.0747 & & 4.2 \\
\hline 130 & 31.45 & 2.8368 & 2.8366 & 3.3 & 310 & 30.41 & 2.9280 & 2.9301 & 68.6 \\
\hline-131 & 34.76 & 2.5747 & 2.5756 & 6.1 & 130 & 31.57 & 2.8235 & 2.8238 & 6.4 \\
\hline-202 & 35.03 & 2.5554 & 2.5560 & 4.4 & 221 & 32.35 & 2.7575 & 2.7566 & 7.8 \\
\hline 002 & 36.13 & 2.4804 & 2.4778 & 9.0 & 102 & 34.70 & 2.5766 & 2.5742 & 4.8 \\
\hline 221 & 36.57 & 2.4516 & 2.4509 & 3.0 & 131 & 35.87 & 2.4955 & 2.4980 & 5.6 \\
\hline-231 & 37.18 & 2.4128 & 2.4119 & 1.6 & 202 & 38.67 & 2.3217 & 2.3217 & 2.8 \\
\hline 400 & 39.06 & 2.3013 & 2.3005 & 1.5 & 231 & 39.64 & 2.2672 & 2.2653 & 2.2 \\
\hline 311 & 40.49 & 2.2234 & 2.2234 & 1.5 & 212 & 40.04 & 2.2455 & 2.2464 & 2.3 \\
\hline 102 & 40.63 & 2.2161 & 2.2183 & 1.2 & 330 & 42.06 & 2.1425 & 2.1432 & 6.6 \\
\hline-331 & 41.96 & 2.1490 & 2.1482 & 4.9 & 411 & 43.48 & 2.0760 & 2.0758 & 1.4 \\
\hline 330 & 42.21 & 2.1368 & 2.1381 & 1.8 & 141 & 45.12 & 2.0045 & 2.0047 & 8.7 \\
\hline-421 & 42.58 & 2.1192 & 2.1195 & 1.8 & 331 & 45.45 & 1.9907 & 1.9899 & 2.0 \\
\hline-402 & 44.07 & 2.0510 & 2.0510 & 2.2 & 510 & 49.94 & 1.8222 & 1.8225 & 5.2 \\
\hline 321 & 44.30 & 2.0409 & 2.0422 & 1.5 & 150 & 52.26 & 1.7468 & 1.7465 & 3.4 \\
\hline 041 & 44.34 & 2.0392 & 2.0384 & 1.7 & 431 & 52.73 & 1.7323 & 1.7321 & 1.4 \\
\hline 240 & 44.98 & 2.0117 & 2.0113 & 2.4 & 113 & 53.11 & 1.7209 & 1.7206 & 2.8 \\
\hline-241 & 46.17 & 1.9627 & 1.9635 & 3.3 & 142 & 54.44 & 1.6820 & 1.6823 & 4.3 \\
\hline 510 & 50.52 & 1.8037 & 1.8027 & 6.8 & 023 & 55.33 & 1.6571 & 1.6570 & 7.7 \\
\hline-521 & 51.19 & 1.7816 & 1.7828 & 1.6 & 440 & 57.22 & 1.6068 & 1.6074 & 10.3 \\
\hline 222 & 51.38 & 1.7755 & 1.7757 & 1.5 & 251 & 58.00 & 1.5871 & 1.5865 & 1.0 \\
\hline 150 & 51.98 & 1.7565 & 1.7562 & 2.0 & 600 & 59.44 & 1.5521 & 1.5517 & 2.6 \\
\hline-242 & 54.40 & 1.6841 & 1.6831 & 1.3 & 350 & 59.83 & 1.543 & 1.5428 & 1.2 \\
\hline-223 & 56.49 & 1.6267 & 1.6262 & 11.8 & 531 & 61.17 & 1.5124 & 1.5125 & 4.9 \\
\hline 440 & 57.40 & 1.6030 & 1.6035 & 1.8 & 133 & 61.34 & 1.5086 & 1.5093 & 8.8 \\
\hline 350 & 59.76 & 1.5452 & 1.5454 & 3.4 & 060 & 62.60 & 1.4813 & 1.4817 & 2.6 \\
\hline-233 & 61.48 & 1.5062 & 1.5065 & 2.7 & 152 & 63.54 & 1.4617 & 1.4629 & 0.9 \\
\hline 060 & 62.18 & 1.4909 & 1.4909 & 5.8 & 043 & 67.11 & 1.3925 & 1.3921 & 2.4 \\
\hline-352 & 65.68 & 1.4195 & 1.4199 & 1.1 & 333 & 68.26 & 1.3718 & 1.3719 & 6.7 \\
\hline \multirow[t]{4}{*}{531} & 67.43 & 1.3871 & 1.3871 & 5.3 & 451 & 68.58 & 1.3662 & 1.3662 & 0.7 \\
\hline & & & & & 602 & 69.95 & 1.3428 & 1.3427 & 1.0 \\
\hline & & & & & 631 & 70.62 & 1.3317 & 1.3315 & 5.4 \\
\hline & & & & & 104 & 70.99 & 1.3256 & 1.3257 & 2.4 \\
\hline \multirow{2}{*}{\multicolumn{10}{|c|}{$\begin{array}{l}\text { a These are not equal to the } \mathrm{d} \text { values calculated from the observed } 2 \theta \text { values by the Bragg equation because systematic errors were used } \\
\text { in the least-squares refinements. }\end{array}$}} \\
\hline & & & & & & & & & \\
\hline b) & 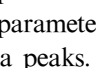 & $a=931$ & 15) $\AA$ & $0(19)$ & 30 & & & & \\
\hline
\end{tabular}

$\mathrm{PEn}$ at $1150^{\circ} \mathrm{C}$ be the origin of these two extra peaks which appeared along with PEn with increasing temperature in all runs. The constant appearance of these two extra peaks implies that they are controlled by the intrinsic structure basis upon heating.

The smaller $d$ values of the two extra peaks at $1200^{\circ} \mathrm{C}$ are $3.075,3.074,3.079$ and $3.079 \AA$ for the first, second, third and fourth runs, respectively, and the larger ones are $3.331,3.323,3.327$ and $3.328 \AA$ for respective runs. The smaller $d$ values correspond to $d=3.06 \AA$ $\left(1145^{\circ} \mathrm{C}\right)$ of the characteristic peak of the new high temperature phase reported by Ernst and Schwab (1970). However, our results are different from theirs in that there was no reappearance of the two extra peaks in the cooling process of PEn as shown in the third and fourth runs. These features suggest that these two extra peaks belong to a metastable phase.

High-T CEn reported by Smith (1969) has the strongest peak of $d=3.06 \AA$ and the strong peak of $d=$ $3.34 \AA$ at $1100^{\circ} \mathrm{C}$ which do not overlap with the peaks of 
Table 3. Refined cell parameters for low clinoenstatite (CEn) and protoenstatite (PEn) at room and high temperatures in the second run

\begin{tabular}{ccccccc}
\hline Phase & Temp. $\left({ }^{\circ} \mathrm{C}\right)$ & $\mathrm{a}(\AA)$ & $\mathrm{b}(\AA)$ & $\mathrm{c}(\AA)$ & $\beta\left(^{\circ}\right)$ & $\mathrm{V}\left(\AA^{3}\right)$ \\
\hline \multirow{6}{*}{ CEn } & 25 & $9.6091(16)$ & $8.8201(13)$ & $5.1726(8)$ & $108.326(8)$ & $416.16(12)$ \\
& 1000 & $9.7197(25)$ & $8.9412(22)$ & $5.2359(14)$ & $108.938(16)$ & $430.40(20)$ \\
& 1100 & $9.7418(19)$ & $8.9544(19)$ & $5.2487(12)$ & $109.031(11)$ & $432.83(17)$ \\
& $25^{*}$ & $9.6099(19)$ & $8.8193(16)$ & $5.1726(10)$ & $108.331(10)$ & $416.15(14)$ \\
& 1200 & $9.3084(22)$ & $8.8847(17)$ & $5.3563(10)$ & & \\
& 1250 & $9.3156(21)$ & $8.8943(17)$ & $5.3589(10)$ & & $442.98(16)$ \\
& 1300 & $9.3176(22)$ & $8.9044(19)$ & $5.3601(10)$ & & $444.02(16)$ \\
& 1350 & $9.3183(24)$ & $8.9097(23)$ & $5.3609(12)$ & & $444.71(16)$ \\
& 1400 & $9.3217(22)$ & $8.9176(18)$ & $5.3627(10)$ & & $445.08(19)$ \\
& 1450 & $9.3316(22)$ & $8.9269(18)$ & $5.3663(11)$ & & $445.79(16)$ \\
& $1400^{*}$ & $9.3298(23)$ & $8.9182(19)$ & $5.3642(11)$ & & $447.02(17)$ \\
& $1350^{*}$ & $9.3220(19)$ & $8.9079(17)$ & $5.3622(10)$ & & $445.27(15)$ \\
& $1300^{*}$ & $9.3170(18)$ & $8.9022(15)$ & $5.3606(10)$ & & $444.62(14)$ \\
& $1250^{*}$ & $9.3147(21)$ & $8.8954(16)$ & $5.3581(10)$ & & $443.96(15)$ \\
& $1200^{*}$ & $9.3129(20)$ & $8.8881(16)$ & $5.3579(10)$ & & $443.49(15)$ \\
& $1100^{*}$ & $9.3021(18)$ & $8.8680(15)$ & $5.3543(8)$ & & $441.68(13)$ \\
& $1000^{*}$ & $9.3009(23)$ & $8.8571(18)$ & $5.3529(11)$ & & $440.97(17)$ \\
\hline
\end{tabular}

*Temperature in the cooling process.

PEn. We can interpret that these strong peaks correspond to our two extra peaks. Although the d-spacings $(3.323-3.331 \AA)$ of the lower $2 \theta$ peak of our two extra peaks at $1200^{\circ} \mathrm{C}$ are slightly smaller than that expected from $3.34 \AA$ at $1100^{\circ} \mathrm{C}$ by $\operatorname{Smith}(1969)$, this may be due to the fact that this peak is overlapped with the neighbouring higher $2 \theta$ peak in our study (Figs. 2 and 3 ) and is slightly shifted toward the higher $2 \theta$ side. Therefore, it will be reasonable to deduce that these two extra peaks originate from the small amount of metastable high-T CEn coexisting with PEn. As shown by the distinct discontinuous change of the cell volume in the CEn-PEn transition in Figure 4, the CEn-PEn transition is the first order and the structure difference between CEn and PEn seems larger than that between CEn and high-T CEn. Therefore, the CEn-PEn transition will be slower than the CEn-high-T CEn transition. Because of this, the small amount of CEn still survived above the CEn-PEn transition temperature, and they inverted to high-T CEn instead of PEn above the metastable transition temperature between CEn and high-T CEn, resulting in the coexistence of the large amount of PEn and the small amount of high-T CEn. Survival of high-T CEn for the wide temperature range above the metastable transition temperature, as observed in Figures 2 and 3 , may indicate that once metastable high-T CEn is formed, it does not easily transform into stable PEn.

\section{Stability field of high-T CEn above PEn ?}

In the fourth run up to $1550^{\circ} \mathrm{C}$ in our study we did not observe any other peak except those of PEn from $1500^{\circ} \mathrm{C}$ to the melting point, indicating PEn is the only existing phase. The abrupt change of peak intensities of PEn at temperatures from $1540^{\circ} \mathrm{C}$ to $1550^{\circ} \mathrm{C}$ and the intensity reduction of the PEn peaks at $1550^{\circ} \mathrm{C}$ with time probably imply that temperature is very close to the melting point and the sample was affected by the precursory effect before melting. Therefore, we may say that there is no stability field for high-T CEn above that of PEn, because there is very little possibility that the transition from PEn to high-T CEn (probably the first order) needs much more time than that of the fourth run $(\sim 3$ hours at $1550^{\circ} \mathrm{C}$ ), or that PEn transforms into high-T $\mathrm{CEn}$ in the very narrow interval of $1550^{\circ} \mathrm{C}-1557^{\circ} \mathrm{C}$. This is consistent with the report by Smyth (1974) that he did not observe high-T CEn above the stability field of PEn till the incongruent melting point.

\section{Stability relations of enstatite polymorphs}

The schematic diagram of the Gibbs free energies of enstatite polymorphs can be estimated from the data of appearances and disappearances of enstatite polymorphs with increasing and decreasing temperature in the pres- 

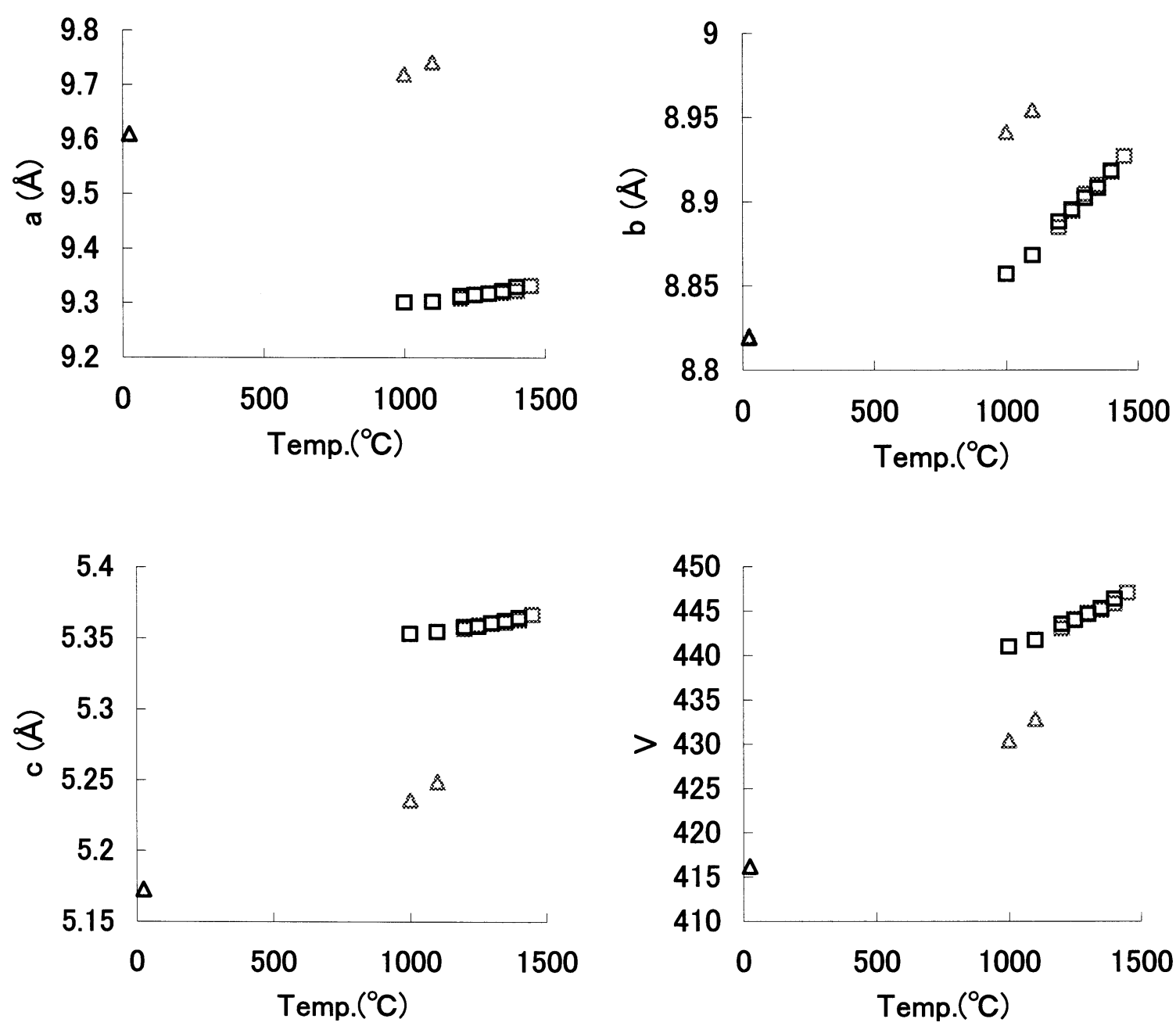

Figure 4. Variations of cell parameters of CEn and PEn with temperature in the second run. Triangle and square symbols represent CEn and PEn, respectively. Symbols with gray line mean the process of increasing temperature and symbols with solid line mean the process of decreasing temperature. Standard deviations are smaller than the symbols.

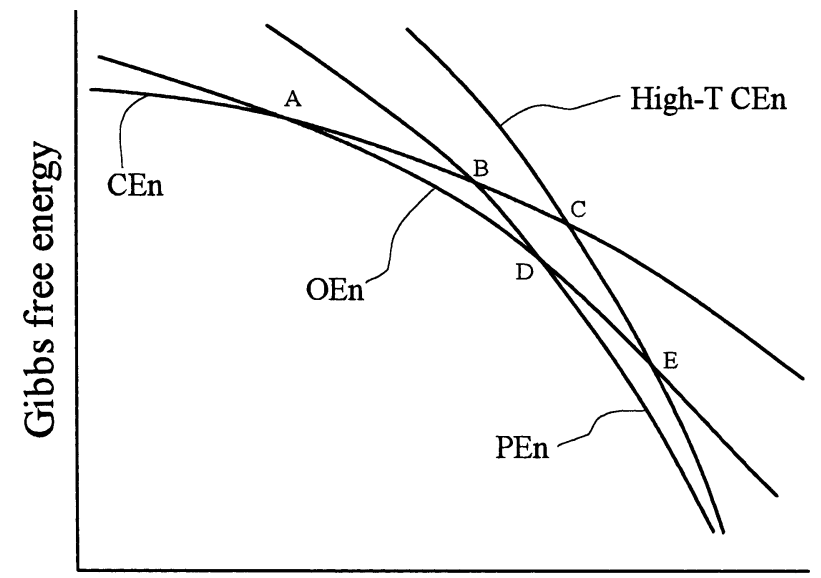

Temperature

Figure 5. Estimated Gibbs free energy diagram for the enstatite polymorphs. ent study, and the results of previous studies, as illustrated in Figure 5. Based on this free energy diagram, the transition behaviors of enstatite polymorphs with the variation of temperature in our high temperature $\mathrm{X}$-ray diffraction experiments are explained as follows.

When CEn is heated from room temperature, OEn becomes stable at temperature above $\mathrm{A}$ in Figure 5. By the sluggish transition from CEn to OEn, however, CEn does not transform into OEn. Even at B, CEn does not instantly transform into PEn because the CEn-PEn transition is the first order and sluggish, although it is not so sluggish as the CEn-OEn one. After some overheating beyond $\mathrm{B}$, most of CEn transforms to PEn and the remaining small amount of CEn transforms metastably into high- $\mathrm{T} C \mathrm{C}$ above $\mathrm{C}$ because of the fast CEn-high-T CEn transition. The coexistence of a large amount of PEn and a small amount of metastable high-T CEn can be explained by these different rates of 
both transformations. With further temperature increase, the small amount of high-T CEn eventually transforms into PEn.

When a mixture of PEn and metastable high-T CEn is cooled as for the first and second runs, high-T CEn inverts into CEn below temperature C. Meanwhile PEn becomes unstable below point $\mathrm{D}$, but does not transform into OEn because of the sluggish transition rate of PEn into OEn. PEn inverts to CEn after some undercooling beyond $\mathrm{B}$, although some part remains as PEn to room temperature depending on the cooling rate. Therefore, on cooling high-T CEn disappears first and then PEn disappears as observed in the first and second runs. When the single phase of PEn is cooled as for the third and fourth runs, PEn passes through point $\mathrm{D}$ and transforms to CEn after some undercooling beyond B. It should be remarked that in this case there is no chance for high-T CEn to appear because the free energy of high-T CEn is always higher than that of PEn. In this way, the observed appearances and disappearances of enstatite polymorphs in all the runs are successfully explained.

From this diagram, transition behaviors of enstatite polymorphs using OEn as a starting material are also predicted. When OEn is used as a starting material, metastable appearance of high-T CEn with increasing temperature will also be possible above E. In this case, high-T CEn will appear at higher temperatures than in the case where CEn is used as a starting material, as observed by Shimobayashi and Kitamura (1993), because of the relative positions of $\mathrm{C}$ and $\mathrm{E}$ on the free energy-temperature curve of high-T CEn.

However, it must be remembered that the actual appearance or disappearance of high-T CEn depends on the kinetic factors such as rate of temperature rise or duration of stay at a particular temperature. This will be the cause of the variations in the appearance of high$\mathrm{T}$ CEn observed in the previous studies.

\section{Concluding remarks}

High temperature in situ X-ray diffraction studies of enstatite $\mathrm{MgSiO}_{3}$ up to the melting point elucidated the following points hitherto controverted.

1. Upon heating, CEn transformed into PEn at around $1100^{\circ} \mathrm{C}$ with a discontinuous change of molar volume, and PEn persisted up to the melting point.

2. Two extra diffraction peaks which could not be indexed as CEn or PEn appeared along with PEn at around $1150^{\circ} \mathrm{C}$ but disappeared above $1400-1500^{\circ} \mathrm{C}$ with further temperature rise. These peaks did not reappear on cooling of PEn. From these behaviors coupled with d-spacings of these peaks, they are thought to be originated from high-T CEn metastably transformed from CEn.

3. PEn is the high temperature stable phase up to the melting point, and there seems no stability field for high-T CEn above and below that of PEn.

\section{Acknowledgments}

This work was supported by Grant-in-Aid for Scientific Research 99034 of the Japan Society for the Promotion of Science. The authors thank two anonymous reviewers who helped to improve the manuscript.

\section{References}

Angel, R.J., Chopelas, A. and Ross, N.L. (1992) Stability of high-density clinoenstatite at upper-mantle pressures. Nature, 358, 322-324.

Boysen, H., Frey, F., Schrader, H. and Eckold, G. (1991) On the proto- to ortho-/clino enstatite phase transformation: Single crystal $\mathrm{x}$-ray and inelastic neutron investigation. Physics and Chemistry of Minerals, 17, 629-635.

Ernst, TH. and Schwab, R. (1970) Stability and structural relations of $(\mathrm{Mg}, \mathrm{Fe})$ Metasilicates. Physics of the Earth and Planetary Interiors, 3, 451-455.

Gasparik, T. (1990) A thermodynamic model for the enstatite-diopside join. American Mineralogist, 75, 10801091.

Iishi, K. and Kitayama, K. (1995) Stability of high clinoenstatite. Neues Jahrbuch für Mineralogie Monatshefte H. 2, $65-74$.

Kushiro, I. (1972) Determination of the liquidus relations in synthetic silicate systems with electron probe analysis: The system forsterite-diopside-silica at 1 atmosphere. American Mineralogist, 57, 1260-1271.

Matsui, M. and Price, G.D. (1992) Computer simulation of the $\mathrm{MgSiO}_{3}$ polymorphs. Physics and Chemistry of Minerals, 18, 365-372.

Murakami, T., Takeuchi, Y. and Yamanaka, T. (1982) The transition of orthoenstatite to protoenstatite and the structure at $1080^{\circ} \mathrm{C}$. Zeitschrift für Kristallographie, 160, 299312.

Pannhorst, W. (1984) High-T crystal structure refinement of low clinoenstatite up to $700^{\circ} \mathrm{C}$. Neues Jahrbuch für Mineralogie Abhandlungen, 150, 3, 219-228.

Perrotta, A.J. and Stephenson, D.A. (1965) Clinoestatite: high-low inversion. Science, 148, 1090-1091.

Sadanaga, R., Okamura, F.P. and Takeda, H. (1969) X-ray study of the phase transformations of enstatite. Mineralogical Journal, 6, 110-130.

Sadanaga, R. and Okamura, F.P. (1971) On the high-clino phase of enstatite. Mineralogical Journal, 6, 365-374.

Sarver, J.F. and Hummel, F.A. (1962) Stability relations of magnesium metasilicate polymorphs. Journal of the American Ceramic Society, 45, 152-157.

Schrader, H., Boysen, H., Frey, F. and Convert, P. (1990) On the phase transformation proto- to clino/orthoenstatite: neutron powder investigations. Physics and Chemistry of 
Minerals, 17, 409-415.

Shimobayashi, N. and Kitamura, M. (1991) Phase transition in $\mathrm{Ca}$-poor clinopyroxenes: a high temperature transmission electron microscopic study. Physics and Chemistry of Minerals, 18, 153-160.

Shimobayashi, N. and Kitamura, M. (1993) Phase transition of orthoenstatite to high-clinoenstatite: in situ TEM study at high temperatures. Mineralogical Journal, 16, 416-426.

Smith, J.V. (1969) Magnesium pyroxene at high temperature : Inversion in clinoenstatite. Nature, 222, 256-257.

Smyth, J.R. (1969) Orthopyroxene-high-low clinopyroxene inversions. Earth and Planetary Science Letters, 6, 406-
407.

Smyth, J.R. (1974) Experimental study on the polymorphism of enstatite. American Mineralogist, 59, 345-352.

Yang, H. and Ghose, S. (1995) High temperature single crystal $\mathrm{X}$-ray diffraction studies of the ortho-proto phase transition in enstatite, $\mathrm{Mg}_{2} \mathrm{Si}_{2} \mathrm{O}_{6}$ at $1360 \mathrm{~K}$. Physics and Chemistry of Minerals, 22, 300-310.

Manuscript received; 17 August, 2001

Manuscript accepted; 7 January, 2002 